\title{
Development of a Process Model for the Vacuum Assisted Resin Transfer Molding Simulation by the Response Surface Method
}

\author{
Chensong (Jonathan) Dong * \\ Rakon Limited \\ One Pacific Rise, Mt. Wellington \\ Private Bag 99943, Newmarket \\ Auckland, New Zealand \\ chensongdong@yahoo.com
}

\begin{abstract}
The vacuum assisted resin transfer molding process (VARTM) offers many advantages over the traditional resin transfer molding such as lower tooling cost, room temperature processing. In the VARTM process, complete filling of the mold with adequate wetting of the fibrous preform is critical to the product quality. Computer simulation has become a powerful tool for liquid composite molding process design and optimization. However, in the VARTM process, since the presence of High Permeable Media (HPM), which has a much higher permeability than fiber, 3-D models are necessary and the extensive computation limits the usage of simulation in the process design and optimization.
\end{abstract}

With the permeability, porosity, and thickness of the fiber preform and the RTM mold filling time as references, the dimensionless VARTM process variables and mold filling time are introduced in this paper. The significant process variables were identified by using the Design of Experiments (DOE). A quadratic regression model was developed by the RSM. The model was validated against the 3-D VARTM simulation

\footnotetext{
* To whom all correspondence should be addressed.
} 
and experiments. The results show that the accuracy is within $15 \%$ for most commonly used cases while the computation time saving is over 99\%. The approach presented in this paper provides a general guideline for the VARTM process design and optimization. The process variables and flow media can be quickly chosen by using the developed regression model, which is extremely useful for composite part design in the early stage.

Keywords: E: resin flow; C: computational modeling; High Permeable Medium (HPM); Response Surface Method (RSM)

\section{Introduction}

The vacuum assisted resin transfer molding process (VARTM) offers many advantages over the traditional resin transfer molding such as lower tooling cost, room temperature processing. This process has been employed to manufacture many large components ranging from turbine blades and boats to rail cars and bridge decks.

The VARTM process can be divided into five steps. First, in pre-molding, the mold surface is cleaned. Then mold release agent and gel coat are sprayed onto the surface. Next, during reinforcement loading, dry fiber mats are mounted into the mold and covered by a flexible bag film. The cavity is sealed, e.g. by vacuum tapes. Vacuum is created in the mold cavity to draw the resin into the fiber mats. After the cavity is filled with resin, the resin cures and solidifies into the composite part. Finally, the solidified composite is taken out of the mold. Although this process appears simple, in actual fabrication, the procedure can be quite complicated. The locations of the inlets and 
outlets must be carefully selected so that the mold can be completely filled. The mold and resin temperature must be monitored to avoid resin gelling during resin infusion.

This study focuses on one of the common VARTM processes: the Seemann Composite Resin Infusion Molding Process (SCRIMP), which was invented and patented in the late 1980’s by Bill Seemann. In this process, a highly permeable distribution medium is incorporated into fiber preform as a surface layer. During infusion, resin flows preferentially across the surface and simultaneously through the preform thickness, which enables large parts to be fabricated.

Complete filling of the mold with adequate wetting of the fibrous preform is critical in the VARTM. Incomplete impregnation in the mold leads to defective parts containing dry spots. In order to achieve good quality, processing parameters such as the locations and numbers of gates and vents need to be properly set.

Traditionally, trial-and-error techniques are widely applied in the composite industry, which largely depend on the experience and skills of operators. It is very costly and time consuming. With the development of computing technology, simulation has become a powerful tool for the process design and optimization. The Control Volume Finite Element Method (CVFEM) has been the predominant method for process simulation [1-6]. It forms and solves a set of equations for nodal control volumes as if they were finite elements. Mesh regeneration is not required, which makes the computation more efficient.

In the conventional RTM process, fiber is the only flow medium. The part can often be regarded as a shell and simulation in 2-D domain. The simulation techniques are quite developed and several commercial simulation software packages are available [7- 
10]. In the VARTM process, due to the existence of two distinct flow media, fiber preform and High Permeable Medium (HPM), usually 3-D models are required for simulation.

When a 3-D model is used, for large parts, the VARTM simulation requires a large number of nodes and elements during meshing. In addition, the distribution medium is usually much thinner than the preform. Therefore, a finer mesh is needed to avoid the high aspect ratio, which may result in poor conditioning in simulation, as well as from discretization errors. This uses a large amount of computer hardware resources and increases the computer load. The simulation time increases significantly and makes the simulation not feasible.

VARTM simulation has been studied extensively. Mathur et al. [11] developed an analytical model, which predicts the flow times and flow front shapes as a function of the properties of the preform, distribution media and resin. Further, they formulated a performance index to give a measure of the process efficacy. Loos et al. [12] developed a 3-D model to simulate the VARTM manufacturing process of complex shape composite structures. Mohan et al. [13] modeled and characterized the flow in channels using equivalent permeability. The equivalent permeability is used as input for numerical simulation of the mold filling process. The numerical simulations are based on a pure finite element based methodology. The mold filling in the VARTM was investigated by Sun et al. [14] based on a High Permeable Medium and Ni [15] et al. based on grooves. A 3-D Control Volume Finite Element Method was adopted to solve the flow governing equations. Based on experimental observations and CVFEM simulation, a simplified leakage flow model was presented, where they considered the preform and the peel ply as 
a sink for the resin, while modeling the flow in the distribution layer. Tari et al. [16] derived a closed form model for vacuum bag resin transfer molding under several simplifying assumptions. They assumed that the resin velocity in the saturated fiber preform is negligible. Hsiao et al. [17] avoided this assumption and hence the velocity for the resin, as well as the shape of the flow front through the thickness of the fiber preform, was accurately captured. Han et al. [18] proposed a hybrid 2.5-D and 3-D flow model. Dong [19] presented an Equivalent Medium Method for improving computation efficiency of the VARTM simulation.

From the literature survey, most of the studies focused on the development of simplified models due to the extensive computations involved in the 3-D CVFEM method. However, the 3-D nature of VARTM simulation is not avoided. Thus, the simulation is more time-consuming than 2-D RTM simulation. In addition, the 3-D Finite Element modeling and material defining process are more complicated due to the anisotropic nature of flow media. The fiber direction needs to be specified for each element to relate the material orientation to the coordinate system, as shown in Figure 2. In view of the industrial application, the mold filling process and time need to be predicted in a timely manner to reduce the lead time. The 3-D simulation is incapable of meeting this requirement. Thus, it is necessary to develop a more convenient tool for VARTM simulation.

Dimensionless VARTM process variables including dimensionless permeability, porosity, and thickness have been introduced in this paper. The dimensionless VARTM mold filling time was also derived by correlating the VARTM process with the RTM process. The significant process variables were identified by using the Design of 
Experiments (DOE). A quadratic regression model was developed by the RSM. The VARTM mold filling time can be obtained by multiplying the RTM mold filling time of the same composite part by the dimensionless VARTM mold filling time. The model was validated against the 3-D VARTM simulation and experiments. The results show that the accuracy is within $10 \%$ for most commonly used cases while the computation time saving is over 99\%. The original contributions of this research are:

1. A guideline for VARTM process design is provided. The process parameters can be selected per this guideline.

2. An efficient and effective approach is developed for the VARTM process optimization. By using this approach, the process parameters can be found to meet the objective in a time-efficient way.

3. Since variations exist for the process parameters [20], the approach presented in this paper can also be used as a robust design tool to reduce the sensitivity to the variations of process parameters.

The rest of the paper is structured as follows. In section 2, the approach to develop a VARTM process regression model is presented. The model validation is presented in Section 3. Section 4 discusses the application of the presented model. Conclusions are drawn in Section 5.

\section{Approach}

\subsection{Control Volume Finite Element Method (CVFEM)}

The flow of a viscous fluid through an anisotropic, homogenous, porous medium is represented by Darcy's law [21]: 


$$
\left\{\begin{array}{l}
u \\
v \\
w
\end{array}\right\}=-(1 / \mu)\left[\begin{array}{lll}
K_{x x} & K_{x y} & K_{x z} \\
K_{y x} & K_{y y} & K_{y z} \\
K_{z x} & K_{z y} & K_{z z}
\end{array}\right]\left\{\begin{array}{l}
\partial p / \partial x \\
\partial p / \partial y \\
\partial p / \partial z
\end{array}\right\}
$$

where $K_{i j}(i, j=x, y$, or $z)$ are the components of the permeability tensor. $\partial p / \partial x, \partial p / \partial y$ and $\partial p / \partial z$ are the pressure gradients in the three directions respectively.

For an incompressible fluid, the mass conservation equation can be reduced to the form:

$$
\partial u / \partial x+\partial v / \partial y+\partial w / \partial z=0 .
$$

Equation 2 can be integrated over a control volume and leads to:

$$
\iiint_{V}(\partial u / \partial x+\partial v / \partial y+\partial w / \partial z) d V=0 .
$$

Using the Divergence theorem (Gauss's theorem), the control volume integral can be transformed into a control surface integral. Thus, Equation 3 can be written as:

$$
\iint_{S}\left\lfloor\begin{array}{lll}
n_{x} & n_{y} & n_{z}
\end{array}\right\}\left\{\begin{array}{l}
u \\
v \\
w
\end{array}\right\} d S=0
$$

where $n_{x}, n_{y}$ and $n_{z}$ are the normal components of the surface vector of the control volume. Substituting Equation 1 into Equation 4 yields:

$$
\iint_{S}(1 / \mu)\left\lfloor n_{x} \quad n_{y} \quad n_{z}=\left[\begin{array}{lll}
K_{x x} & K_{x y} & K_{x z} \\
K_{y x} & K_{y y} & K_{y z} \\
K_{z x} & K_{z y} & K_{z z}
\end{array}\right]\left\{\begin{array}{l}
\partial p / \partial x \\
\partial p / \partial y \\
\partial p / \partial z
\end{array}\right\} d S=0 .\right.
$$

Equation 5 is the working equation for solving the problems of flow through anisotropic porous media and is a combination of the mass and momentum equations, while the momentum equation is represented by using the Darcy's law. 
In order to solve such moving boundary problems as the resin flow front advances using the traditional finite element method, it requires the computation domain redefinition and mesh regeneration. Mesh regeneration needs a large amount of computation time as the domain becomes complicated. Alternatively, the control volume finite element method, which forms and solves a set of equations for nodal control volumes as if they were finite elements, does not require mesh regeneration. Thus, the computation is more efficient.

The boundary conditions for mold filling simulation are as follows:

At the flow front:

$$
p=0 .
$$

At the inlet gates:

For constant pressure: $p=p_{0}$;

For the constant flow rate: $\mathbf{v}=\mathbf{v}_{0}$.

At the mold boundaries:

$$
\partial p / \partial \mathbf{n}=0 .
$$

It is assumed that at the beginning of mold filling, the control volumes enclosing the inlet nodes are filled with resin. At the flow front, a parameter $f$ is used to represent the status of each control volume in the flow domain. If the control volume has not been occupied by the fluid, $f$ is equal to zero. If the control volume is partially filled, $f$ is equal to the volume fraction of the fluid occupying the control volume. $f$ factor is set to 1 if the volume is completely filled by advancing fluid. The control volumes with $f$ values varying between 0 and 1 are considered flow front elements. The pressure in these partially filled flow front control volumes is set to the ambient pressure. With the 
aforementioned boundary conditions, the set of linear algebraic equations can be solved to determine the pressure field at each time step during mold filling. Based on the calculated pressure field, the velocity field can then be computed using Darcy's law.

The time increment is selected in such a way that a control volume will be fill at each time step. Sometimes, several control volumes can be filled simultaneously. After $f$ values are updated, another pressure computation is performed for all the fully filled control volumes. The process is repeated until the whole mold is filled [2].

\subsection{RTM and VARTM Simulation}

In the traditional RTM process, fiber preform is the only flow medium. The through-thickness resin flow can often be neglected and thus a 2-D model can be applied. In the VARTM process, however, another flow medium — High Permeable Medium (HPM) presents. Considering a 1-D flow in the VARTM process, the resin flow front is

plotted in Figure 2. The HPM is much thinner than the preform. The flow is assumed to be well developed and can be divided into two regions: saturated region and flow front region. In the saturated region, no cross-flow exists, while in the flow front region the resin is infiltrating into the preform from the HPM.

When the inputs in the CVFEM mold filling simulation are considered, they can be divided into:

- Geometric properties: molding geometry

- Material properties: permeability and porosity

- Condition properties: injection method and pressure, viscosity of resin The closed form solution for the 1-D flow through a homogeneous fiber preform can be derived from Darcy's law as 


$$
t=l^{2} \phi \mu /\left(2 p_{0} K\right)
$$

Expanded to the general case, it can be derived that

$$
t \propto \phi \mu /\left(p_{0} K\right) .
$$

If the reference variables are $\phi_{\text {ref, }}, \mu_{\text {ref, }} p_{0 \text { ref }}$ and $K_{\text {ref, }}$ and the reference mold filling time is $t_{\text {ref, }}$ Equation 11 can be written as

$$
t=t_{\text {ref }}\left[\phi \mu /\left(p_{0} K\right)\right] /\left[\phi_{\text {ref }} \mu_{\text {ref }} /\left(p_{\text {oref }} K_{\text {ref }}\right)\right]
$$

When the material properties of fiber are considered as the only variables, Equation 12 becomes

$$
t=t_{\text {ref }}(\phi / K) /\left(\phi_{\text {ref }} / K_{\text {ref }}\right)
$$

In the VARTM process, two types of flow media - fiber preform and HPM exist. If the properties of fiber preforms and the RTM mold filling time are regarded as the reference variables and mold filling time, respectively, the VARTM mold filling time can be derived by using Equation 13 as

$$
\left.t_{\text {VARTM }}=t_{\text {RTM }} \mid F_{1}\left(\phi_{f}, \phi_{H}, h_{f}, h_{H}\right) / F_{2}\left(K_{f}, K_{H}, h_{f}, h_{H}\right)\right] /\left(\phi_{f} / K_{f}\right)
$$

Equation 14 shows that the VARTM mold filling time is dependent on the permeability and porosity of fiber preform and HPM, and the composition of fiber preform and HPM. It can be further written as

$$
t_{\text {VARTM }}=t_{\text {RTM }}\left\lfloor G_{1}\left(\phi_{H} / \phi_{f}, h_{H} / h_{f}\right) / G_{2}\left(K_{H} / K_{f}, h_{H} / h_{f}\right)\right\rfloor
$$

If the dimensionless process variables for the VARTM process are derived as $K^{*}=K_{H} / K_{f}, \phi^{*}=\phi_{H} / \phi_{f}, h^{*}=h_{H} / h_{f}$, and the dimensionless VARTM mold filling time is given by the VARTM-RTM mold filling time ratio as $t^{*}=t_{\mathrm{VARTM}} / t_{\mathrm{RTM}}$, Equation 15 becomes 


$$
t_{\mathrm{VARTM}}=t_{\mathrm{RTM}}\left[G_{1}\left(\phi^{*}, h^{*}\right) / G_{2}\left(K^{*}, h^{*}\right)\right]
$$

As a preliminary study, several RTM and VARTM mold filling process simulation for the same part by both linear and port injection was simulated. For RTM simulation, 2-D models were used and for VARTM simulation 3-D models were used. The permeability was considered as the only variable. The thickness is $4 \mathrm{~mm}$ for the fiber preform and $1 \mathrm{~mm}$ for the HPM. The porosity is 0.5 for the fiber preform and 0.8 for the HPM. The injection pressure is $1 \times 10^{5} \mathrm{~Pa}$. When the permeability of the fiber preform and HPM is 100 Darcy and 3,000 Darcy, respectively, the RTM and VARTM mold filling process is shown in Figure 4. The complete result for various permeability values is shown in Table 1. Thus, it is confirmed that the dimensionless mold filling time is dependent on $\phi^{*}, h^{*}$ and $K^{*}$. For a VARTM manufacturer, the microscopic level through-thickness flow is usually of less importance than the in-plane resin flow front development and mold filling time. Thus, it is possible to use 2-D RTM simulation and dimensionless VARTM process variables to simulate the mold filling time.

The approach is illustrated in Figure 5. For any given composite part design, the mold filling process can be simulated in 2-D using the RTM assumption. The dimensionless VARTM process variables $\phi^{*}, h^{*}$ and $K^{*}$ are calculated and the dimensionless VARTM mold filling time $t^{*}$ is calculated by the developed VARTM process model. The actual VARTM mold filling time can be given by

$$
t_{\mathrm{VARTM}}=t^{*} t_{\mathrm{RTM}} .
$$




\subsection{Screening Design}

First, a screening design was conducted to uncover the individual contributions to the VARTM mold filling time of $h_{\mathrm{H}}, \phi_{\mathrm{H}}$ and $K_{\mathrm{H}}$. A factorial design was conducted to identify the significant factors.

Normally, the permeability is 50 200 Darcy for fiber preforms and 1,000 5,000 Darcy for HPM. The porosity is $0.4 \sim 0.6$ for fiber preforms and 0.7 0.9 for HPM. The thickness of HPM is less than $1 \mathrm{~mm}$. In order to derive the dimensionless variables, $K_{\mathrm{f}}$, $\phi_{\mathrm{f}}$ and $h_{\mathrm{f}}$ were fixed at 100 Darcy, 0.5 , and $4 \mathrm{~mm}$, respectively. The levels of $h_{\mathrm{H}}, \phi_{\mathrm{H}}$ and $K_{\mathrm{H}}$ are shown in Table 2. These were chosen to cover the normal application range. The dimensionless VARTM process variables were derived and the dimensionless VARTM mold filling time is the response.

A full $2^{3}$ factorial design with center points were chosen. After data analysis, the half normal plot, the main effects and interaction plots are shown in Figure 6. It shows that the significant factors are: $h^{*}, K^{*}$, and the interaction of $h^{*}$ and $K^{*}$. The ANOVA also indicates that a significant curvature exists.

\subsection{Model Development by Response Surface Method (RSM)}

The result from the screening design shows that $\phi^{*}$ is an insignificant variable so that only $h^{*}, K^{*}$ are used in the further analysis.

The significant effect of curvature indicates that a linear model is not sufficient. A second-order response surface model needs to be developed. One effective way to develop such a model is the Central Composite Design (CCD). Thus, the design was augmented into a $2^{2}$ design with four axial runs as shown in Figure 7. 
The Box-Cox Method [22] was used to stabilize the variance. An inverse transformation of the flow time was chosen. The final fitted regression model is

$$
t^{*}=1 /\left(a_{0}+a_{1} h^{*}+a_{2} K^{*}+a_{11} h^{* 2}+a_{22} K^{* 2}+a_{12} h^{*} K^{*}\right)
$$

where

$$
\begin{aligned}
& a_{0}=-1.51 \times 10^{-1} \\
& a_{1}=8.62 \\
& a_{2}=4.50 \times 10^{-2} \\
& a_{11}=-1.84 \times 10^{1} \\
& a_{22}=-5.35 \times 10^{-5} \\
& a_{12}=5.01 \times 10^{-1}
\end{aligned}
$$

Its corresponding response surface is shown in Figure 8.

\section{Model Validation}

A boat hull as shown in Figure 9 was simulated to validate the regression model. Its length is $2 \mathrm{~m}$.

The permeability is 80 Darcy for the fiber preform and 3,000 Darcy for the HPM. The porosity is 0.5 for the fiber preform and 0.9 for the HPM the porosity.

First, the thickness of the fiber preform was assumed to be $20 \mathrm{~mm}$. The processing of the boat hull by the RTM process was simulated by using a 2-D CVFEM model. Half of the structure was modeled due to its symmetry, as shown in Figure 10(a). The injection pressure was $1 \times 10^{5} \mathrm{~Pa}$. The viscosity was $200 \mathrm{cP}$. The mold filling process is shown in Figure 10(b). The mold filling time is 13,903 sec.

When the VARTM processing of the boat hull was considered, the thickness of the HPM was assumed to be $1 \mathrm{~mm}$. The dimensionless variables were calculated as 
$h^{*}=0.05$

$K^{*}=37.5$.

The mold filling time was calculated as

$$
t_{\mathrm{VARTM}}=t^{*} t_{\mathrm{RTM}}=4,993 \mathrm{~s} .
$$

The VARTM mold filling process was also simulated by using a 3-D CVFEM model. The result is shown in Figure 11 . The mold filling time is 5,597 s. The computation time is $2 \mathrm{~h} 50$ '. The relative error is $11 \%$ and the time saving is $>99 \%$.

Secondly, the thickness of the fiber preform was assumed to be $10 \mathrm{~mm}$. The mold filling time from simulation is 13,903 sec. When the VARTM processing of the boat hull was considered, the thickness of the HPM was also assumed to be $1 \mathrm{~mm}$. The dimensionless variables were calculated as

$$
\begin{aligned}
& h^{*}=0.1 ; \\
& K^{*}=37.5 .
\end{aligned}
$$

The mold filling time was calculated as

$$
t_{\mathrm{VARTM}}=t^{*} t_{\mathrm{RTM}}=3,461 \mathrm{~s} .
$$

The mold filling time from simulation is 3,608 s. The computation time is $1 \mathrm{~h} 41$ '. The relative error is $4 \%$ and the time saving is $>99 \%$.

The developed model was further experimentally validated through the VARTM processing of a car hood. The approximate dimensions are: 1,300 $\mathrm{mm} \times 900 \mathrm{~mm}$. The permeability and porosity was determined from measurement as $K_{f}=90$ Darcy, $\phi_{f}=0.52, K_{H}=500$ Darcy, $\phi_{H}=0.82$. The resin was injected from the center of the hood at the pressure of 0.6 vacuum and the viscosity was $500 \mathrm{cP}$. The mold filling 
process simulated by the 2-D CVFEM is shown in Figure 12. The RTM mold filling time is $4,545 \mathrm{~s}$. The thickness of the fiber preform and the HPM is $2.5 \mathrm{~mm}$ and $0.65 \mathrm{~mm}$, respectively. Thus, the dimensionless variables can be derived as $h^{*}=0.26$ and $K^{*}=5.56$. The dimensionless VARTM mold filling time can be calculated as $t^{*}=0.55$

and the VARTM mold filling time can be calculated as $t_{\mathrm{VARTM}}=2,500 \mathrm{~s}$. The actual mold filling process is shown in Figure 13. The mold filing time was 2,910 s. The relative error is $14 \%$.

\section{Model Application}

In the Liquid Composite Molding, the viscosity increases with increasing curing time and temperature. The rate of viscosity increase is low at the early stages of curing. After a threshold degree of cure is achieved, the resin viscosity increases at a very rapid rate. The time at which this occurs is called the gel time. It is necessary to complete the mold filling process before the gel time since the flow of resin in the mold becomes increasingly difficult at the end of this time period.

From the modeling by the RSM, a general HPM selection chart has been developed as shown in Figure 14. The horizontal and vertical axes are dimensionless thickness and permeability, respectively, and the contour is the dimensionless mold filling time. This chart can assist in selecting the HPM to achieve desirable mold filling time.

As an example, from 2-D simulation we know that the $t_{\mathrm{RTM}}=80 \mathrm{~min}$. The gel time of the resin being used is $30 \mathrm{~min}$. Thus, it is desirable that $t_{\mathrm{VARTM}} \leq 25 \mathrm{~min}$, i.e. 


$$
t^{*}=t_{\mathrm{VARTM}} / t_{\mathrm{RTM}} \leq 0.31 \text {. }
$$

Thus, the dimensionless thickness and permeability should be selected from the shaded area shown in Figure 15. The corresponding thickness and permeability of the HPM can be easily calculated.

\section{Conclusions}

In this paper, a VARTM process model developed by 2-D CVFEM simulation, the Design of Experiments, and the Response Surface Method (RSM) is presented. With the permeability, porosity, and thickness of the fiber preform and the RTM mold filling time as references, the dimensionless VARTM process variables and mold filling time are introduced in this paper. The significant process variables were identified by using the Design of Experiments (DOE). A quadratic regression model was developed by the RSM. The model was validated against the 3-D VARTM simulation and experiments. The results show that the accuracy is within $15 \%$ for most commonly used cases while the computation time saving is over 99\%. The approach presented in this paper provides a general guideline for the VARTM process design and optimization. The process variables and flow media can be quickly chosen by using the developed regression model, which is extremely useful for composite part design in the early stage. It is also noticed that for thick composite parts, this model yields a larger error because of the neglecting of the through-thickness resin flow.

The advantages of this model are that the computation time is reduced significantly. The complicated 3-D Finite Element modeling and material property defining process are avoided, which makes this model very convenient for practical 
applications. For example, the mold filling time can be obtained promptly when the permeability and/or the thickness of the HPM have changed. The range for developing the RSM based regression model have covered the normal application range. Thus, the model is adequate in predicting the mold filling time of the VARTM process. The HPM selection chart presented in this paper provides a convenient tool for flow media selection and process optimization, which is extremely useful for composite part design in the early stage.

The original contributions of this research are:

1. A guideline for VARTM process design is provided. The process parameters can be selected per this guideline.

2. An efficient and effective approach is developed for the VARTM process optimization. By using this approach, the process parameters can be found to meet the objective in a time-efficient way.

3. Since variations exist for the process parameters, the approach presented in this paper can also be used as a robust design tool to reduce the sensitivity to the variations of process parameters.

\section{References}

[1] Bruschke MV, Advani SG. Filling simulation of complex three-dimensional shelllike structures. SAMPE Quarterly 1991; 23(1): 2-11.

[2] Young WB, Han K, Fong LH, Lee LJ. Flow simulation in molds with preplaced fiber mats. Polymer Composites 1991; 12(6): 391-404. 
[3] Liu XL. Isothermal flow simulation of liquid composite molding. Composites Part A 2000; 31(12): 1295-1302.

[4] Lin RJ, Lee LJ, Liou MJ. Non-isothermal mold filling and curing simulation in thin cavities with preplaced fiber mats, International Polymer Processing 1991; VI(4): 356-369.

[5] Young WB. Three-dimensional nonisothermal mold filling simulation in resin transfer molding. Polymer Composites 1994; 15(2): 118-127.

[6] Lee LJ, Young WB, Lin RJ. Mold filling and curing modeling of RTM and SCRIM processes. Composite Structures 1994; 27(1-2): 109-120.

[7] Simacek P., Advani SG. Desirable features in mold filling simulations for liquid molding processes. Polymer Composites (2004); 25(4): 355-367.

[8] Bruschke MV, Advani SG. A finite element/control volume approach to mold filling in anisotropic porous media. Polymer Composites 1990; 11(6): 398-405.

[9] ESI Group. PAM-RTM. www.esi-group.com.

[10] Polyworx. www.polyworx.com.

[11] Mathur R, Heider D, Hoffmann C, Gillespie JW Jr., Advani SG, Fink BK. Flow front measurements and model validation in the vacuum assisted resin transfer molding process. Polymer Composites 2001; 22(4): 477-490.

[12] Loos AC, Sayre J, McGrane R, Grimsley B. VARTM process model development. In: Proceedings of International SAMPE Symposium and Exhibition. Long Beach, CA, May, 2001. 46(I): 1049-1061.

[13] Mohan RV, Shires DR, Tamma KK, Ngo ND. Flow channels/fiber impregnation studies for the process modeling/analysis of complex engineering structures 
manufactured by resin transfer molding. Polymer Composites 1998; 19(5): 527542.

[14] Sun XD, Li SJ, Lee LJ. Mold filling analysis in vacuum-assisted resin transfer molding. part I: SCRIMP based on a high-permeable medium. Polymer Composites 1998; 19(6): 807-817.

[15] Ni J, Li SJ, Sun XD, Lee LJ. Mold filling analysis in vacuum-assisted resin transfer molding. Part II: SCRIMP based on grooves. Polymer Composites 1998; 19(6): 818-829.

[16] Tari MJ, Imbert JP, Lin MY, Lavine AS, Hahn HT. Analysis of resin transfer molding with high permeability layers. Transactions of the ASME, Journal of Manufacturing Science and Engineering 1998; 120(3): 609-616.

[17] Hsiao KT, Mathur R, Advani SG. A closed form solution for flow during the vacuum assisted resin transfer molding process. Transactions of the ASME, Journal of Manufacturing Science and Engineering 2000; 122(3): 463-475.

[18] Han K, Jiang SL, Zhang C, Wang B. Flow modeling and simulation of SCRIMP for composite manufacturing. Composites Part A 2000; 31(1): 79-86.

[19] Dong CS. An equivalent medium method for the vacuum assisted resin transfer molding process simulation. To appear in Journal of Composite Materials.

[20] Dong CS. Uncertainty analysis for fiber permeability measurement. Transactions of the ASME, Journal of Manufacturing Science and Engineering 2005; 127(4).

[21] D’Arcy H. Les Fontaines Publiques de la villa de Dijon. Paris: Dalmont, 1856.

[22] Box GEP, Cox DR. An analysis of transformations, Journal of the Royal Statistical Society, B 1964; 26: 211-243. 


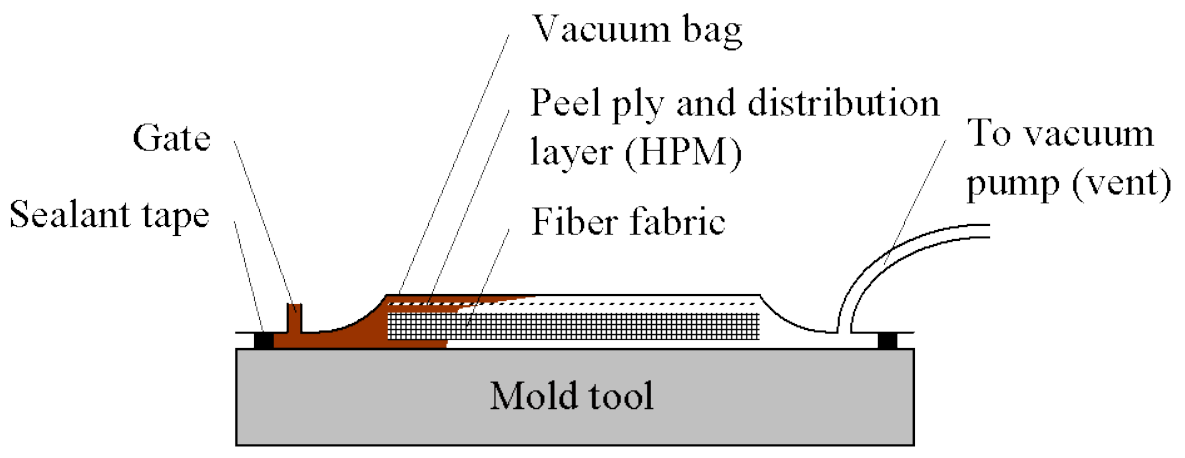

Figure 1: Vacuum assisted resin transfer molding process

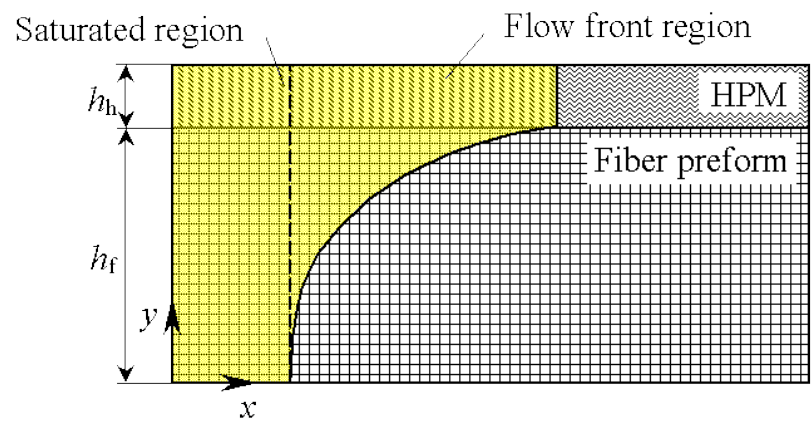

Figure 2: Resin flow in VARTM process 


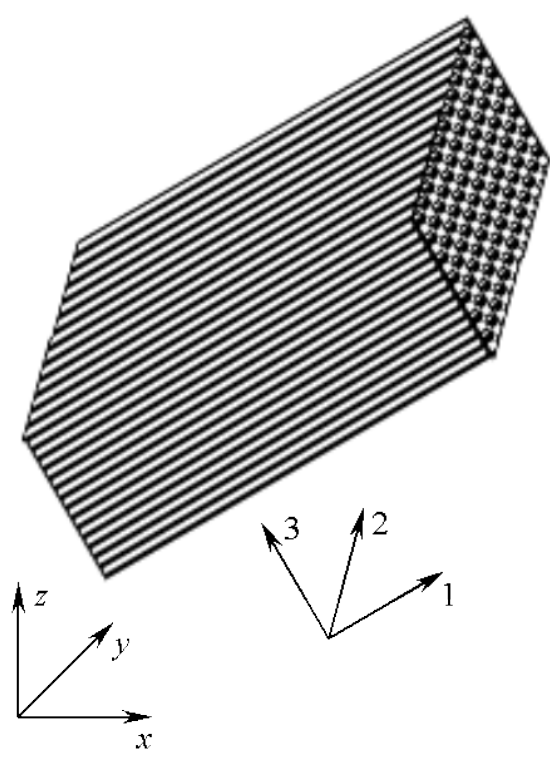

Figure 3: 3-D anisotropy of composites
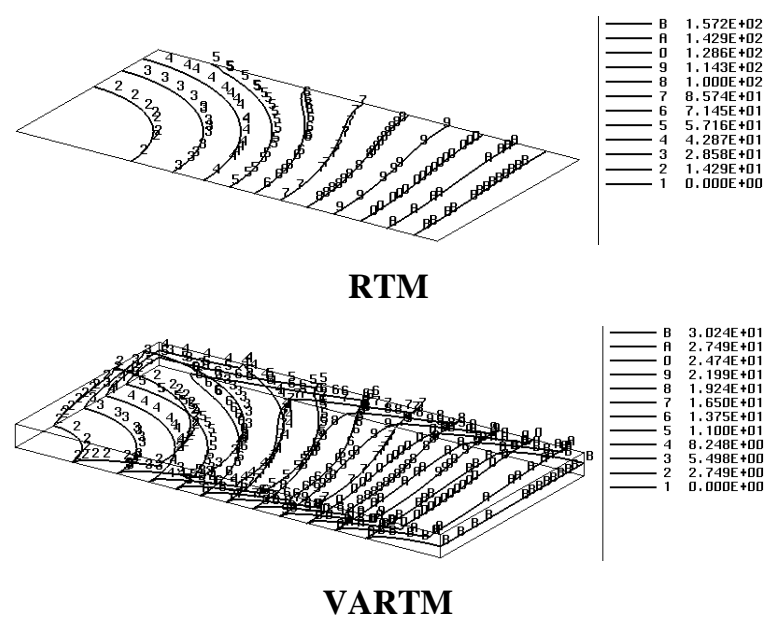

Figure 4: RTM vs. VARTM mold filling 


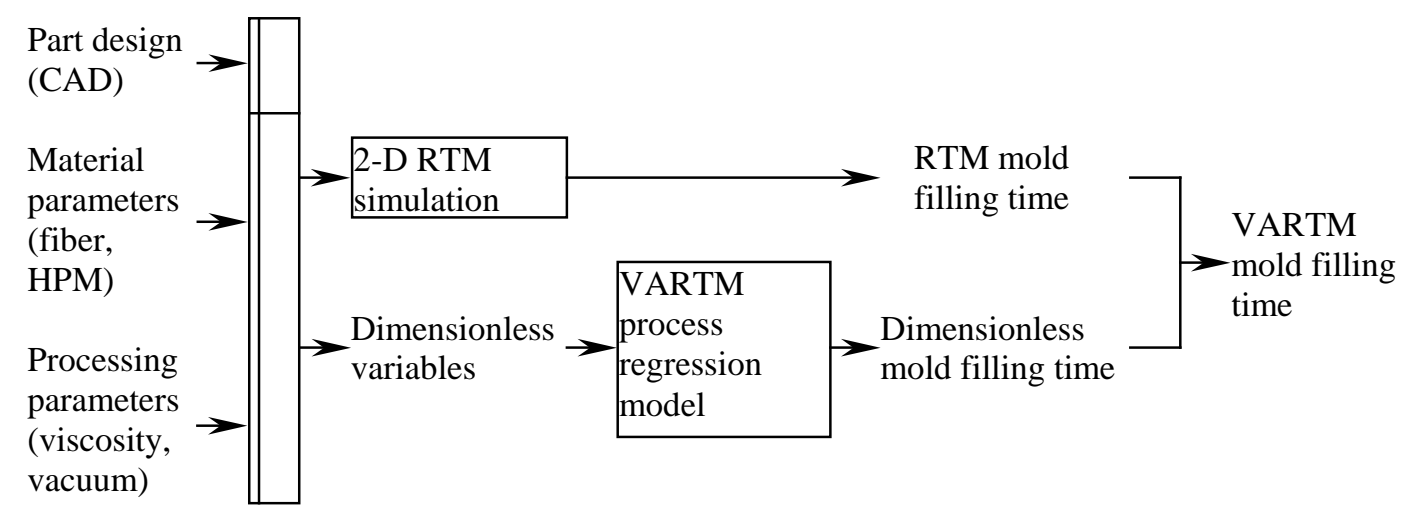

Figure 5: Approach flowchart

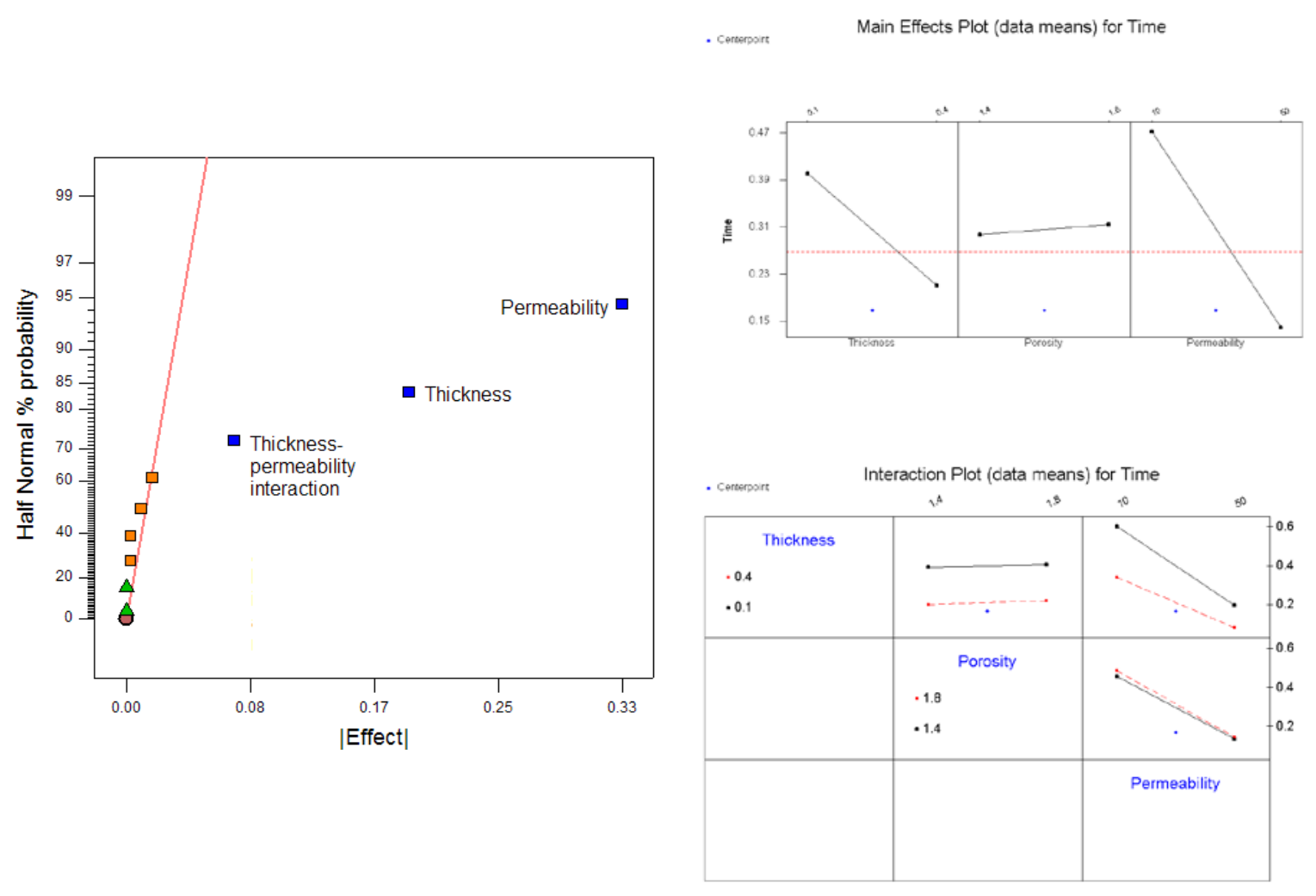

Figure 6: Half normal plot, main effects and interaction plots 


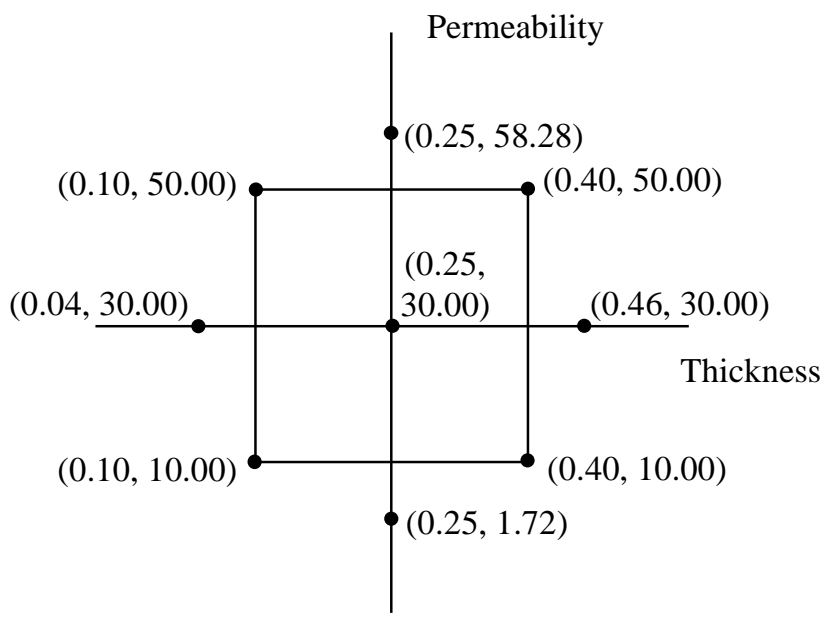

Figure 7: Central Composite Design (CCD)

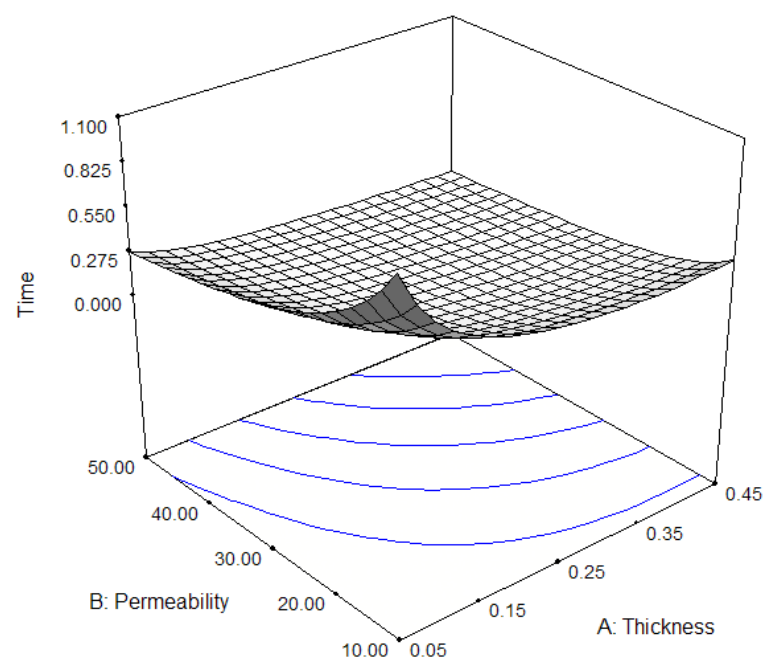

Figure 8: Response surface of the fitted regression model

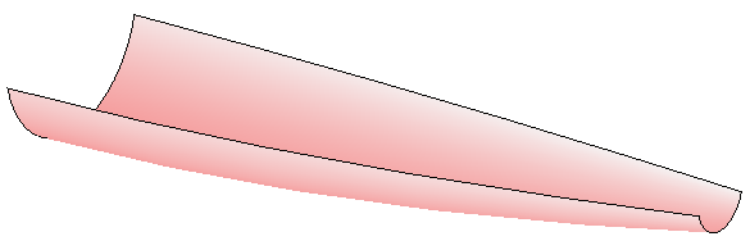

Figure 9: A boat hull 


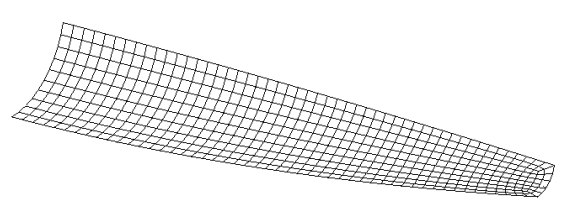

(a) Mesh

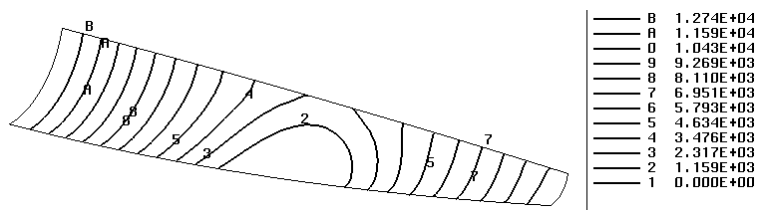

(b) Resin flow

Figure 10: RTM boat hull processing

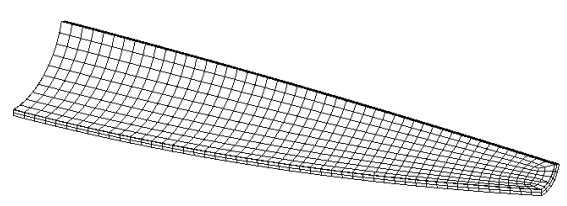

a) Mesh

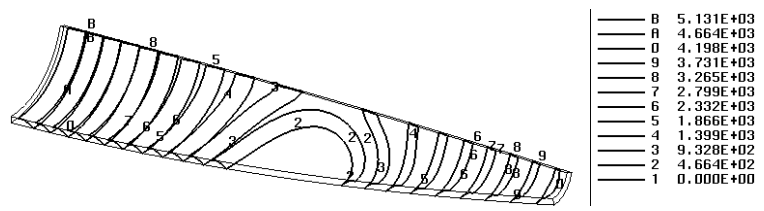

b) Resin flow

Figure 11: VARTM boat hull processing
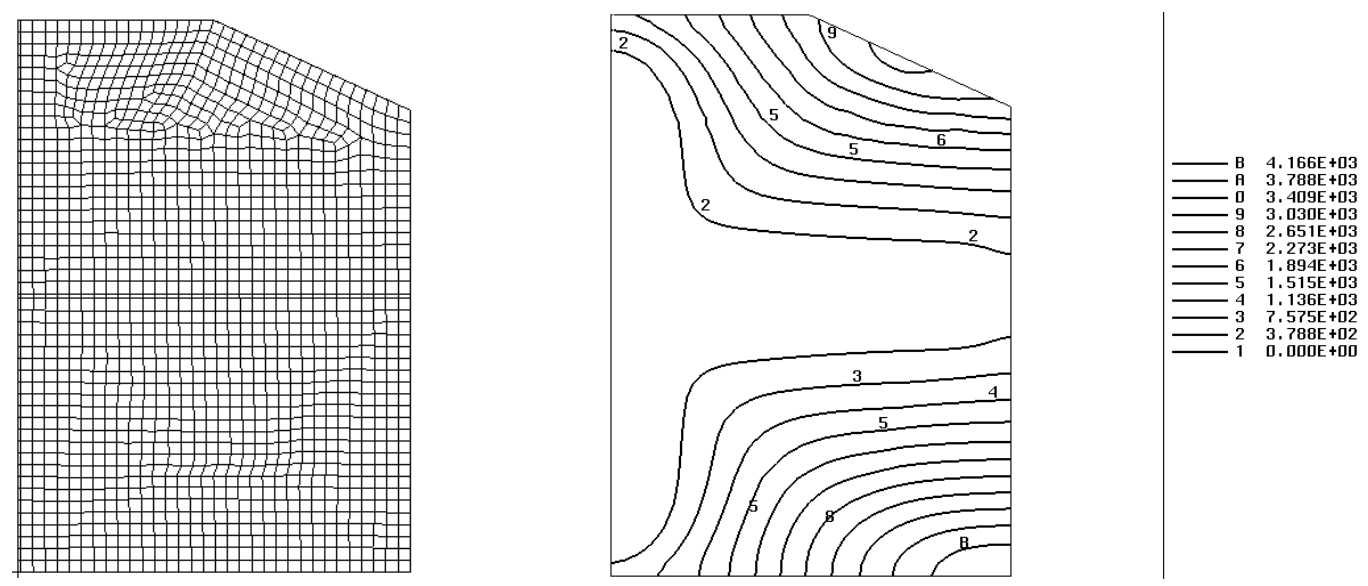

Figure 12: RTM simulation of car hood 


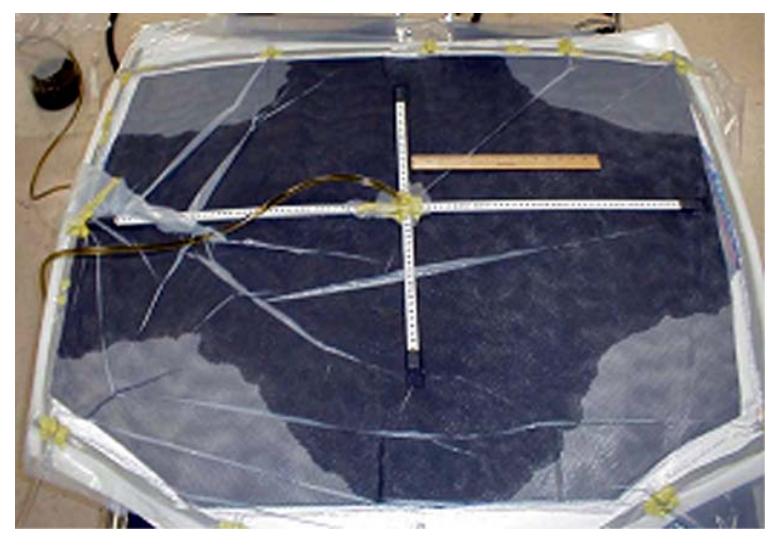

Figure 13: VARTM processing of car hood

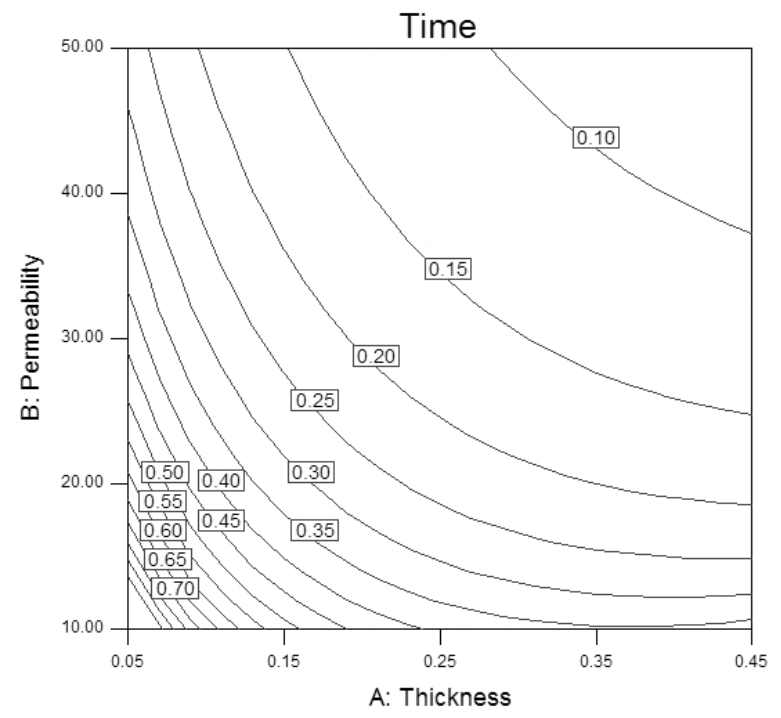

Figure 14: General HPM selection chart 


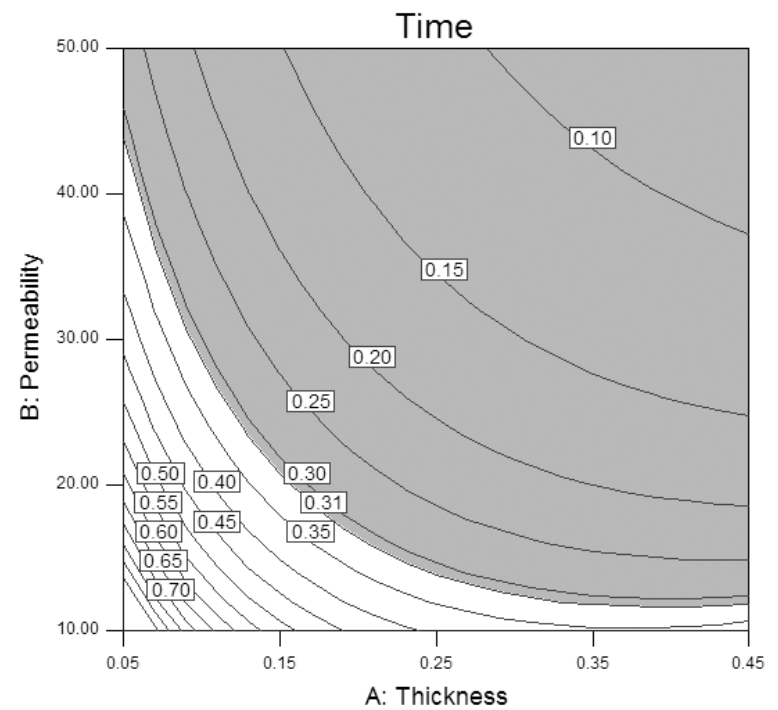

Figure 15: Example of HPM selection 
Table 1: Mold filling simulation of RTM and VARTM processes

\begin{tabular}{|l|l|l|l|l|l|}
\hline & \multicolumn{2}{|l|}{ Permeability (darcy) } & \multicolumn{2}{l|}{ Mold filling time (s) } & \multirow{2}{*}{$\begin{array}{l}\text { VARTM-RTM mold } \\
\text { filling time ratio }\end{array}$} \\
\hline & $K_{11 \mathrm{f}}$ & $K_{11 \mathrm{H}}$ & RTM & VARTM & 0.170 \\
\hline Linear & 50 & 1500 & 98.90 & 16.80 & 0.170 \\
\hline & 100 & 3000 & 49.50 & 8.41 & 0.170 \\
\hline & 200 & 6000 & 24.70 & 4.20 & 0.170 \\
\hline Port & 50 & 1500 & 343.00 & 66.00 & 0.192 \\
\hline & 100 & 3000 & 171.00 & 33.00 & 0.193 \\
\hline & 200 & 6000 & 85.70 & 16.50 & 0.193 \\
\hline
\end{tabular}

Table 2: Level selection

\begin{tabular}{|l|l|l|l|l|l|}
\hline & Low & High & & Low & High \\
\hline$h_{\mathrm{H}}(\mathrm{mm})$ & 0.4 & 1.6 & $h^{*}$ & 0.1 & 0.4 \\
\hline$\phi_{\mathrm{H}}$ & 0.7 & 0.9 & $\phi^{*}$ & 1.4 & 1.8 \\
\hline$K_{\mathrm{H}}($ darcy $)$ & 1,000 & 5,000 & $K^{*}$ & 10 & 50 \\
\hline
\end{tabular}

